\title{
Travelling Wave Solutions for Three Dimensional Incompressible MHD Equations
}

\author{
M. Aldhabani ${ }^{1,2}$, S. M. Sayed ${ }^{3}$ \\ ${ }^{1}$ School of Mathematical, RMIT University, Melbourne, Australia \\ ${ }^{2}$ Mathematics Department, Faculty of Science, University of Tabouk (UT), Tabouk, KSA \\ ${ }^{3}$ Mathematics Department, Faculty of Science, Beni-Suef University, Beni-Suef, Egypt \\ Email: msaed.2@hotmail.com, smsayed71@gmail.com
}

How to cite this paper: Aldhabani, M. and Sayed, S.M. (2018) Travelling Wave Solutions for Three Dimensional Incompressible MHD Equations. Journal of Applied Mathematics and Physics, 6, 114-121. https://doi.org/10.4236/jamp.2018.61011

Received: November 6, 2017

Accepted: January 9, 2018

Published: January 12, 2018

Copyright $\odot 2018$ by authors and Scientific Research Publishing Inc. This work is licensed under the Creative Commons Attribution International License (CC BY 4.0).

http://creativecommons.org/licenses/by/4.0/

\begin{abstract}
In this paper, the solutions of three dimensional incompressible magnetohydrodynamics (MHD) equations are obtained by using $\sin (k \xi)-\cos (k \xi)(\xi=\xi(x, y, z, t), k \geq 0)$ method and Riccati auxiliary equation. This paper obtains the soliton solutions by the aid of software Mathematica.
\end{abstract}

\section{Keywords}

Incompressible MHD Equations, Riccati Auxiliary Equation, Travelling Wave Solutions

\section{Introduction}

The word MHD is made up of three terms magneto indicating magnetic field, hydro referring liquid, and dynamics meaning movement. The field of MHD is a fascinatingly rich field of physics and applied mathematics that considers the behavior of an electrically conducting fluid in the presence of an external electromagnetic field. Although inspiring in its own right, MHD also has numerous engineering and science applications. These range from the pursuit of reliable energy sources such as nuclear fusion [1] to understanding near-earth plasmas such as the solar wind [2] and more exotic astrophysical objects such as stars [3], black holes [4], and the interstellar medium [5]. Virtually all of these areas experience the phenomenon of turbulence and the role turbulence places in engineering and science applications are certainly critical. As previously 
mentioned, MHD is concerned with the behavior of fluids in the presence of an external electromagnetic field. Of course, if the fluid does not conduct electricity, then it will not influence, nor will it be influenced by, the external electromagnetic field. Although Faraday initiated the field of MHD, the mathematical formulation came later only after the discovery of Maxwell's equations [6]. Since there are a vast amount of driving forces in the MHD equations, there is a possibility for different types of waves that would propagate through the plasma. In incompressible MHD, we touch on differences between ideal versus (visco-)resistive evolutions [7]. We point out kinematic and full MHD insights into magnetic field amplification. The Elsasser formulation of the governing equations allows to generalize insights from linear to nonlinear wave package behavior, which prominently appear in MHD turbulence theories. We again restrict ourselves to dimensional reasoning to introduce the various scaling laws predicted for the energy spectra. High resolution (pseudo-)spectral simulations have given important clues to the anisotropic nature of MHD turbulence. We also discuss numerical evidence for singular structure growth in incompressible MHD and for small-scale dynamo action, by summarizing selected simulation-based studies. We end this section with an introduction to compressible MHD, where the linear wave picture is richer and allows wave steepening, paving the way to shock-dominated plasma behavior [8].

The MHD description governs the large-scale dynamics of plasmas, and applies to many laboratory as well as astrophysical configurations. Incompressible MHD has traditionally focused on topics like MHD turbulence, dynamo aspects, and singular structure formation. We inspect what kind of waves that can exist through linearization of the MHD equations in parallel with applying Fourier transforms. As a reminder, the incompressible MHD equations are

$$
\begin{gathered}
\nabla \cdot \underline{V}=\nabla \cdot \underline{B}=0, \\
\underline{V}_{t}+(\underline{V} \cdot \nabla) \underline{V}-(\underline{B} \cdot \nabla) \underline{B}+\nabla\left(\underline{P}+12|\underline{B}|^{2}\right) \\
-v_{1} \underline{V}_{x x}-v_{2} \underline{V}_{y y}-v_{3} \underline{V}_{z z}=0, \\
\underline{B}_{t}+(\underline{V} \cdot \nabla) \underline{B}-(\underline{B} \cdot \nabla) \underline{V} \\
-\eta_{1} \underline{B}_{x x}-\eta_{2} \underline{B}_{y y}-\eta_{3} \underline{B}_{z z}=0,
\end{gathered}
$$

where

$$
\begin{gathered}
\underline{V}=\left(V_{1}(x, y, z, t), V_{2}(x, y, z, t), V_{3}(x, y, z, t)\right)^{\mathrm{T}}, \\
\underline{B}=\left(B_{1}(x, y, z, t), B_{2}(x, y, z, t), B_{3}(x, y, z, t)\right)^{\mathrm{T}} \text { and } \underline{P}=\underline{P}(x, y, z, t)
\end{gathered}
$$

represent the unknown velocity field, the magnetic field and the pressure of the flow, respectively, and $v_{1}, v_{2}, v_{3}, \eta_{1}, \eta_{2}$ and $\eta_{3}$ are the viscosity coefficients of the flow. The field of incompressible MHD is a particularly rich subset of physics and applied mathematics. The challenges inherent in the equations provide a plethora of research opportunities. Aside from purely academic pursuits, MHD 
also plays an important role in the development of engineering technologies. Designing suitable engineering systems using electrically conducting fluids requires using computational techniques.

One of the most prominent reasons for this difficulty is the phenomenon of fluid turbulence which again rears its head in MHD [9] [10]. In addition to the velocity field displaying disordered behavior the external electromagnetic field quantities also display such behavior [11]. The main aim of this paper is to use the travelling wave method in the construction of exact soliton solutions for three dimensional incompressible MHD equations.

This paper is organized as follows. In Section 1 we review the main governing equations of incompressible MHD. In Section 2, the $\sin (k \xi)-\cos (k \xi)$ method and the exact solutions for the incompressible MHD problem are presented. Finally, Section 3 contains the conclusion.

\section{Travelling Wave Solutions}

The three dimensional incompressible MHD system (1)-(3) in the scalar form

$$
\begin{aligned}
& V_{1, x}+V_{2, y}+V_{3, z}=B_{1, x}+B_{2, y}+B_{3, z}=0 \\
& V_{1, t}+V_{1} V_{1, x}+V_{2} V_{1, y}+V_{3} V_{1, z}+P_{x}+B_{2}\left(B_{2, x}-B_{1, y}\right) \\
& +B_{3}\left(B_{3, x}-B_{1, z}\right)-v_{1} V_{1, x x}-v_{2} V_{1, y y}-v_{3} V_{1, z z}=0 \\
& V_{2, t}+V_{1} V_{2, x}+V_{2} V_{2, y}+V_{3} V_{2, z}+P_{y}+B_{1}\left(B_{1, y}-B_{2, x}\right) \\
& +B_{3}\left(B_{3, y}-B_{2, z}\right)-v_{1} V_{2, x x}-v_{2} V_{2, y y}-v_{3} V_{2, z z}=0 \\
& V_{3, t}+V_{1} V_{3, x}+V_{2} V_{3, y}+V_{3} V_{3, z}+P_{z}+B_{1}\left(B_{1, z}-B_{3, x}\right) \\
& +B_{2}\left(B_{2, z}-B_{3, y}\right)-v_{1} V_{3, x x}-v_{2} V_{3, y y}-v_{3} V_{3, z z}=0 \\
& B_{1, t}+V_{1} B_{1, x}+V_{2} B_{1, y}+V_{3} B_{1, z}-B_{1} V_{1, x}-B_{2} V_{1, y}-B_{3} V_{1, z} \\
& -\eta_{1} B_{1, x x}-\eta_{2} B_{1, y y}-\eta_{3} B_{1, z z}=0, \\
& B_{2, t}+V_{1} B_{2, x}+V_{2} B_{2, y}+V_{3} B_{2, z}-B_{1} V_{2, x}-B_{2} V_{2, y}-B_{3} V_{2, z} \\
& -\eta_{1} B_{2, x x}-\eta_{2} B_{2, y y}-\eta_{3} B_{2, z z}=0 \\
& B_{3, t}+V_{1} B_{3, x}+V_{2} B_{3, y}+V_{3} B_{3, z}-B_{1} V_{3, x}-B_{2} V_{3, y}-B_{3} V_{3, z} \\
& -\eta_{1} B_{3, x x}-\eta_{2} B_{3, y y}-\eta_{3} B_{3, z z}=0 .
\end{aligned}
$$

To find the travelling wave solution for Equations (4)-(10), we take the transformation

$$
V_{i}(x, y, z, t)=v_{i}(\xi), B_{i}(x, y, z, t)=b_{i}(\xi), P(x, y, z, t)=p(\xi), i=1,2,3
$$

where $\xi=x+y+\alpha z+\beta t$, and change the Equations (4)-(10) into the following ordinary differential equations

$$
\begin{gathered}
v_{1}^{\prime}+v_{2}^{\prime}+\alpha v_{3}^{\prime}=b_{1}^{\prime}+b_{2}^{\prime}+\alpha b_{3}^{\prime}=0, \\
\left(\beta+v_{1}+v_{2}+\alpha v_{3}\right) v_{1}^{\prime}+p^{\prime}+b_{2}\left(b_{2}^{\prime}-b_{1}^{\prime}\right) \\
+b_{3}\left(b_{3}^{\prime}-\alpha b_{1}^{\prime}\right)-\left(v_{1}+v_{2}+\alpha^{2} v_{3}\right) v_{1}^{\prime \prime}=0,
\end{gathered}
$$




$$
\begin{gathered}
\left(\beta+v_{1}+v_{2}+\alpha v_{3}\right) v_{2}^{\prime}+p^{\prime}+b_{1}\left(b_{1}^{\prime}-b_{2}^{\prime}\right) \\
+b_{3}\left(b_{3}^{\prime}-\alpha b_{2}^{\prime}\right)-\left(v_{1}+v_{2}+\alpha^{2} v_{3}\right) v_{2}^{\prime \prime}=0, \\
\left(\beta+v_{1}+v_{2}+\alpha v_{3}\right) v_{3}^{\prime}+\alpha p^{\prime}+b_{1}\left(\alpha b_{1}^{\prime}-b_{3}^{\prime}\right) \\
+b_{2}\left(\alpha b_{2}^{\prime}-b_{3}^{\prime}\right)-\left(v_{1}+v_{2}+\alpha^{2} v_{3}\right) v_{3}^{\prime \prime}=0, \\
\left(\beta+v_{1}+v_{2}+\alpha v_{3}\right) b_{1}^{\prime}-\left(b_{1}+b_{2}+\alpha b_{3}\right) v_{1}^{\prime}-\left(\eta_{1}+\eta_{2}+\alpha^{2} \eta_{3}\right) b_{1}^{\prime \prime}=0, \\
\left(\beta+v_{1}+v_{2}+\alpha v_{3}\right) b_{2}^{\prime}-\left(b_{1}+b_{2}+\alpha b_{3}\right) v_{2}^{\prime}-\left(\eta_{1}+\eta_{2}+\alpha^{2} \eta_{3}\right) b_{2}^{\prime \prime}=0, \\
\left(\beta+v_{1}+v_{2}+\alpha v_{3}\right) b_{3}^{\prime}-\left(b_{1}+b_{2}+\alpha b_{3}\right) v_{3}^{\prime}-\left(\eta_{1}+\eta_{2}+\alpha^{2} \eta_{3}\right) b_{3}^{\prime \prime}=0,
\end{gathered}
$$

where ' $=\frac{\mathrm{d}}{\mathrm{d} \xi}$ balancing the highest order of linear terms with nonlinear terms in the system (12)-(18) suggests the following ansatz

$$
\begin{gathered}
v_{1}=\gamma_{1}+\gamma_{2} \psi, v_{2}=\gamma_{3}+\gamma_{4} \psi, v_{3}=\gamma_{5}+\gamma_{6} \psi, \\
b_{1}=\delta_{1}+\delta_{2} \psi, b_{2}=\delta_{3}+\delta_{4} \psi, b_{3}=\delta_{5}+\delta_{6} \psi, \\
p=\rho_{1}+\rho_{2} \psi+\rho_{3} \psi^{2},
\end{gathered}
$$

where $\gamma_{i}, \delta_{i}, \rho_{i}, i=1,2,3, \alpha$ and $\beta$ are constants to be determined, and the function $\psi$ satisfying a Riccati equation

$$
\psi^{\prime 2}+\varepsilon k^{2} \psi^{2}=\epsilon k^{4}, \quad k \geq 0, \epsilon= \pm 1
$$

then we obtain three kinds of general solutions [12]-[16]

$$
\begin{gathered}
\psi=k \sin k \xi \text { or } \psi=k \cos k \xi \text {, when } \epsilon=1, \\
\psi=\text { constant, when } k=0, \\
\psi=k \cosh k \xi \text {, when } \epsilon=-1 .
\end{gathered}
$$

Substituting (19)-(21) into system (12)-(18) and using the Riccati equation (22), we obtain [17]

$$
\begin{gathered}
\left(\gamma_{2}+\gamma_{4}+\alpha \gamma_{6}\right) \psi^{\prime}=\left(\delta_{2}+\delta_{4}+\alpha \delta_{6}\right) \psi^{\prime}=0 \\
\left(\gamma_{2} \beta\right) \psi^{\prime}+\left(\gamma_{1}+\gamma_{2} \psi\right)\left(\gamma_{2} \psi^{\prime}\right)+\left(\gamma_{3}+\gamma_{4} \psi\right)\left(\gamma_{2} \psi^{\prime}\right) \\
+\alpha\left(\gamma_{5}+\gamma_{6} \psi\right)\left(\gamma_{2} \psi^{\prime}\right)+\left(\rho_{2} \psi^{\prime}\right)+\left(2 \rho_{3} \psi \psi^{\prime}\right)+\left(\delta_{3}+\delta_{4} \psi\right)\left(\delta_{4}-\delta_{2}\right) \psi^{\prime} \\
+\left(\delta_{5}+\delta_{6} \psi\right)\left(\delta_{6}-\alpha \delta_{2}\right) \psi^{\prime}-\left(v_{1}+v_{2}+\alpha^{2} v_{3}\right)\left(\gamma_{2} \psi^{\prime \prime}\right)=0 \\
\left(\beta+\gamma_{1}+\gamma_{2} \psi+\gamma_{3}+\gamma_{4} \psi+\alpha \gamma_{5}+\alpha \gamma_{6} \psi\right)\left(\gamma_{4} \psi^{\prime}\right) \\
+\left(\rho_{2} \psi^{\prime}\right)+\left(2 \rho_{3} \psi \psi^{\prime}\right)+\left(\delta_{1}+\delta_{2} \psi\right)\left(\delta_{2}-\delta_{4}\right) \psi^{\prime} \\
+\left(\delta_{5}+\delta_{6} \psi\right)\left(\delta_{6}-\alpha \delta_{4}\right) \psi^{\prime}-\left(v_{1}+v_{2}+\alpha^{2} v_{3}\right)\left(\gamma_{4} \psi^{\prime \prime}\right)=0 \\
\left(\beta+\gamma_{1}+\gamma_{2} \psi+\gamma_{3}+\gamma_{4} \psi+\alpha \gamma_{5}+\alpha \gamma_{6} \psi\right)\left(\gamma_{6} \psi^{\prime}\right) \\
+\left(\alpha \rho_{2} \psi^{\prime}\right)+\left(2 \alpha \rho_{3} \psi \psi^{\prime}\right)+\left(\delta_{1}+\delta_{2} \psi\right)\left(\alpha \delta_{2}-\delta_{6}\right) \psi^{\prime} \\
+\left(\delta_{3}+\delta_{4} \psi\right)\left(\alpha \delta_{4}-\delta_{6}\right) \psi^{\prime}-\left(v_{1}+v_{2}+\alpha^{2} v_{3}\right)\left(\gamma_{6} \psi^{\prime \prime}\right)=0 \\
\left(\beta+\gamma_{1}+\gamma_{2} \psi+\gamma_{3}+\gamma_{4} \psi+\alpha \gamma_{5}+\alpha \gamma_{6} \psi\right)\left(\delta_{2} \psi^{\prime}\right) \\
-\left(\delta_{1}+\delta_{2} \psi+\delta_{3}+\delta_{4} \psi+\alpha \delta_{5}+\alpha \delta_{6} \psi\right)\left(\gamma_{2} \psi^{\prime}\right) \\
-\left(\eta_{1}+\eta_{2}+\alpha^{2} \eta_{3}\right)\left(\delta_{2} \psi^{\prime \prime}\right)=0
\end{gathered}
$$




$$
\begin{aligned}
& \left(\beta+\gamma_{1}+\gamma_{2} \psi+\gamma_{3}+\gamma_{4} \psi+\alpha \gamma_{5}+\alpha \gamma_{6} \psi\right)\left(\delta_{4} \psi^{\prime}\right) \\
& -\left(\delta_{1}+\delta_{2} \psi+\delta_{3}+\delta_{4} \psi+\alpha \delta_{5}+\alpha \delta_{6} \psi\right)\left(\gamma_{4} \psi^{\prime}\right) \\
& -\left(\eta_{1}+\eta_{2}+\alpha^{2} \eta_{3}\right)\left(\delta_{4} \psi^{\prime \prime}\right)=0, \\
& \left(\beta+\gamma_{1}+\gamma_{2} \psi+\gamma_{3}+\gamma_{4} \psi+\alpha \gamma_{5}+\alpha \gamma_{6} \psi\right)\left(\delta_{6} \psi^{\prime}\right) \\
& -\left(\delta_{1}+\delta_{2} \psi+\delta_{3}+\delta_{4} \psi+\alpha \delta_{5}+\alpha \delta_{6} \psi\right)\left(\gamma_{6} \psi^{\prime}\right) \\
& -\left(\eta_{1}+\eta_{2}+\alpha^{2} \eta_{3}\right)\left(\delta_{6} \psi^{\prime \prime}\right)=0 .
\end{aligned}
$$

Then setting the coefficients of all powers of $\psi, \psi^{\prime}$ and $\psi \psi^{\prime}$ to zero, we will get a set of algebraic system with respect to variables $\gamma_{i}, \delta_{i}, \rho_{i}, i=1,2,3, \alpha$ and $\beta$

$$
\begin{gathered}
\left(\gamma_{2}+\gamma_{4}+\alpha \gamma_{6}\right)=0, \quad\left(\delta_{2}+\delta_{4}+\alpha \delta_{6}\right)=0, \\
\left(v_{1}+v_{2}+\alpha^{2} v_{3}\right)\left(\gamma_{2} k^{2}\right)=0, \\
\gamma_{2} \beta+\gamma_{1} \gamma_{2}+\gamma_{3} \gamma_{2}+\alpha \gamma_{5} \gamma_{2}+\rho_{2}+\delta_{3} \delta_{4}-\delta_{3} \delta_{2}+\delta_{5} \delta_{6}-\alpha \delta_{5} \delta_{2}=0, \\
\gamma_{2}^{2}+\gamma_{2} \gamma_{4}+\alpha \gamma_{6} \gamma_{2}+2 \rho_{3}+\delta_{4}^{2}-\delta_{4} \delta_{2}+\delta_{6}^{2}-\alpha \delta_{6} \delta_{2}=0, \\
\left(v_{1}+v_{2}+\alpha^{2} v_{3}\right)\left(\gamma_{4} k^{2}\right)=0, \\
\gamma_{4} \beta+\gamma_{1} \gamma_{4}+\gamma_{3} \gamma_{4}+\alpha \gamma_{5} \gamma_{4}+\rho_{2}+\delta_{1} \delta_{2}-\delta_{1} \delta_{4}+\delta_{5} \delta_{6}-\alpha \delta_{5} \delta_{4}=0, \\
\gamma_{4}^{2}+\gamma_{2} \gamma_{4}+\alpha \gamma_{4} \gamma_{6}+2 \rho_{3}+\delta_{2}^{2}-\delta_{4} \delta_{2}+\delta_{6}^{2}-\alpha \delta_{6} \delta_{4}=0, \\
\left(v_{1}+v_{2}+\alpha^{2} v_{3}\right)\left(\gamma_{6} k^{2}\right)=0, \\
\gamma_{6} \beta+\gamma_{1} \gamma_{6}+\gamma_{3} \gamma_{6}+\alpha \gamma_{5} \gamma_{6}+\alpha \rho_{2}+\alpha \delta_{1} \delta_{2}-\delta_{1} \delta_{6}+\alpha \delta_{3} \delta_{4}-\delta_{3} \delta_{6}=0, \\
\alpha \gamma_{6}^{2}+\gamma_{2} \gamma_{6}+\gamma_{4} \gamma_{6}+2 \alpha \rho_{3}+\alpha \delta_{2}^{2}-\delta_{6} \delta_{2}+\alpha \delta_{4}^{2}-\delta_{6} \delta_{4}=0, \\
\left(\eta_{1}+\eta_{2}+\alpha^{2} \eta_{3}\right)\left(\delta_{2} k^{2}\right)=0, \\
\delta_{2} \beta+\gamma_{1} \delta_{2}+\gamma_{3} \delta_{2}+\alpha \gamma_{5} \delta_{2}-\gamma_{2} \delta_{1}-\gamma_{2} \delta_{3}-\alpha \delta_{5} \gamma_{2}=0, \\
\gamma_{2} \delta_{2}+\gamma_{4} \delta_{2}+\alpha \gamma_{6} \delta_{2}-\gamma_{2} \delta_{2}-\delta_{4} \gamma_{2}-\alpha \delta_{6} \gamma_{2}=0, \\
\left(\eta_{1}+\eta_{2}+\alpha \eta_{3}^{2}\right)\left(\delta_{4} k^{2}\right)=0, \\
\gamma_{2} \delta_{6}+\gamma_{4} \delta_{6}+\alpha \gamma_{6} \delta_{6}-\gamma_{6} \delta_{2}-\delta_{4} \gamma_{6}-\alpha \delta_{6} \gamma_{6}=0 . \\
\delta_{4} \beta+\gamma_{1} \delta_{4}+\gamma_{3} \delta_{4}+\alpha \gamma_{5} \delta_{4}-\gamma_{4} \delta_{1}-\gamma_{4} \delta_{3}-\alpha \delta_{5} \gamma_{4}=0, \\
\gamma_{2} \delta_{4}+\gamma_{4} \delta_{4}+\alpha \gamma_{6} \delta_{4}-\gamma_{4} \delta_{2}-\delta_{4} \gamma_{4}-\alpha \delta_{6} \gamma_{4}=0, \\
\left(\eta_{1}+\eta_{2}+\alpha^{2} \eta_{3}\right)\left(\delta_{6} k^{2}\right)=0,
\end{gathered}
$$

From the output of symbolic computation software Mathematica, we obtain a solution, namely,

$$
\begin{aligned}
& v_{1}=v_{2}=-2 v_{3}, \quad \eta_{1}=\eta_{2}=-2 \eta_{3}, \\
& \alpha=2, \gamma_{2}=\gamma_{4}=\delta_{2}=\delta_{4}=-\gamma_{6}=-\delta_{6}=d_{0}, \\
& \rho_{3}=\frac{-3}{2} d_{0}^{2}, \quad \rho_{2}=d_{0}\left(3-c_{0}\right), \quad \beta=c_{0}-a_{0},
\end{aligned}
$$

where 


$$
a_{0}=\gamma_{1}+\gamma_{3}+2 \gamma_{5}, \quad c_{0}=\delta_{1}+\delta_{3}+2 \delta_{5},
$$

where $a_{0}, c_{0}, \rho_{2}, \rho_{3}$ and $k$ are arbitrary constants. Since $k$ is a arbitrary parameter, according to (19)-(21), (23)-(25) and (52), we obtain three kinds of travelling wave solutions for the new coupled MHD system (1)-(3), namely

1) a periodic solution with $\epsilon=1$

$$
\begin{gathered}
V_{1}=\gamma_{1}+k d_{0} \sin k \xi, V_{2}=\gamma_{3}+k d_{0} \sin k \xi, V_{3}=\gamma_{5}-k d_{0} \sin k \xi, \\
B_{1}=\delta_{1}+k d_{0} \sin k \xi, B_{2}=\delta_{3}+k d_{0} \sin k \xi, B_{3}=\delta_{5}-k d_{0} \sin k \xi, \\
P=\rho_{1}+k d_{0}\left(3-c_{0}\right) \sin k \xi-\frac{3}{2} d_{0}^{2} k^{2} \sin ^{2} k \xi,
\end{gathered}
$$

2) a soliton solution with $\epsilon=-1$

$$
\begin{gathered}
V_{1}=\gamma_{1}+k d_{0} \cosh k \xi, V_{2}=\gamma_{3}+k d_{0} \cosh k \xi, V_{3}=\gamma_{5}-k d_{0} \cosh k \xi, \\
B_{1}=\delta_{1}+k d_{0} \cosh k \xi, B_{2}=\delta_{3}+k d_{0} \cosh k \xi, B_{3}=\delta_{5}-k d_{0} \cosh k \xi, \\
P=\rho_{1}+k d_{0}\left(3-c_{0}\right) \cosh k \xi-\frac{3}{2} d_{0}^{2} k^{2} \cosh ^{2} k \xi,
\end{gathered}
$$

3) a constant solution with $k=0$

$$
\begin{array}{ccc}
V_{1}=\gamma_{1}, & V_{2}=\gamma_{3}, & V_{3}=\gamma_{5}, \\
B_{1}=\delta_{1}, & B_{2}=\delta_{3}, & B_{3}=\delta_{5}, \\
& P=\rho_{1},
\end{array}
$$

where $\xi=x+y+2 z+\left(c_{0}-a_{0}\right) t$. The MHD equations govern the dynamics of the velocity and the magnetic field in electrically-conducting fluids and reflect the basic physics laws of conservation. These equations can be implemented to study various problems in plasma, liquid metals, saltwater as well as astrophysics. The MHD equations involve coupling between the incompressible Navier-Stokes equations (when the magnetic field $\underline{B}$ is identically equal to 0 ) governing the fluid and incompressible Euler equations for $\underline{B}=0, v_{1}, v_{2}, v_{3}=0$. This paper examines the soliton solutions for the three-dimensional incompressible MHD equations with only magnetic diffusion (without velocity dissipation). MHD deals with the dynamics of an electrically conducting fluid under the influence of magnetic field. The magnetic field, which is present everywhere in the universe, generates magnetic force and this force influences the dynamics of moving fluid, potentially changing the geometry or strength of magnetic field itself. It has been found that the difference in the phase may occur between speed and fluctuations of the magnetic field when the kinetic and magnetic Reynolds numbers are very large. Since the speed and fluctuations of the magnetic field in a circular polarized, the phase difference makes them no longer parallel or anti-parallel like that in the incompressible MHD.

\section{Conclusion}

This paper presents stabilized exact soliton solutions for the incompressible MHD equations. These stabilized soliton solutions are focused at incompressible fluids and the main technological applications in mind are those related with 
material processing techniques. The flow considered here is incompressible and parallel to the magnetic filed. Several classes of soliton solutions are obtained in three-dimensional Cartesian coordinates. Previously, Neukirch [18] obtained self-consistent three-dimensional exact solutions of the MHD equations and solved the basic nonlinear equation in terms of Jacobi elliptic functions. Petrie and Neukirch [19] used a transformation method to study equilibria of the MHD equations and solved the problem vanishing one component of the magnetic field. Petrie et al. [20] obtained two-dimensional exact solutions of MHD equations with application to solar prominences. Here, we have obtained three-dimensional exact solutions in the presence of mass flow and all the three components of the magnetic field. We can discuss the possible applications of some of the obtained solutions to flow phenomena of solar prominences.

\section{References}

[1] Fan, J.H. and Ozawa, T. (2014) Regularity Criteria for the 2D MHD System with Horizontal Dissipation and Horizontal Magnetic Diffusion. Kinetic and Related Models, 7, 45-56. https://doi.org/10.3934/krm.2014.7.45

[2] Ye, Z. (2014) Two Regularity Criteria to the 2D Generalized MHD Equations with Zero Magnetic Diffusivity. Journal of Mathematical Analysis and Applications, 420, 954-971.

http://www.ingentaconnect.com/content/el/0022247x/2014/00000420/00000002 https://doi.org/10.1016/j.jmaa.2014.06.041

[3] Li, Y. (2001) Lax Pair for the 2D Euler Equation. Journal of Applied Mathematics, 42, 3552-3564. https://faculty.missouri.edu/liyan/Lax.pdf https://doi.org/10.1063/1.1378305

[4] Li, Y. and Yurov, A.V. (2003) Lax Pairs and Darboux Transformations for Euler Equations. Studies in Applied Mathematics, 111, 101-113. http://onlinelibrary.wiley.com/doi/10.1111/1467-9590.t01-1-00229/epdf

[5] Zelik, S. (2007) Spatially Nondecaying Solutions of 2D Navier-Stokes Equations in a Strip. Glasgow Mathematical Journal, 49, 525-588.

https://www.researchgate.net/publication/231955261 https://doi.org/10.1017/S0017089507003849

[6] Tran, C.V., Yu, X. and Zhai, Z. (2013) On Global Regularity of 2D Generalized Magnetohydrodynamics Equations. Journal of Differential Equations, 254, 4194-4216. http://www.sciencedirect.com/science/article/pii/S0022039613000922

[7] Klingenberg, W. (1982) Riemannian Geometry. Walter de Gruyter, Berlin, New York.

https://www.amazon.com/Riemannian-Geometry-Degruyter-Studies-Math-ematics /dp/3110145936 https://doi.org/10.1016/j.jde.2013.02.016

[8] Chuu-Lian, T. and Uhlenbeck, K. (1999) Introduction to Surveys in Differential Geometry: Integrable Systems. Journal of Differential Geometry, 4, 5-21. http://intlpress.com/site/pub/pages/books/items/00000109/index.html

[9] Luen Cheung, K. (2014) Exact Solutions for the Two-Dimensional Incompressible Magnetohydrodynamics Equations. Applied Mathematical Sciences, 8, 5915-5922. http://www.m-hikari.com/ams/ams-2014/ams-117-120-2014/cheungAMS117-120-2 014.pdf 
[10] Sayed, S.M. (2013) The Bäcklund Transformations, Exact Solutions, and Conservation Laws for the Compound Modified Korteweg-de Vries-Sine-Gordon Equations which Describe Pseudospherical Surfaces. Journal of Applied Mathematics, 2013, 1-10. https://doi.org/10.1155/2013/613065

[11] Sayed, S.M., Elkholy, A.M. and Gharib, G.M. (2008) Exact Solutions and Conservation Laws for Ibragimov-Shabat Equation Which Describe Pseudo-Spherical Surface. Computational \& Applied Mathematics, 27, 305-318.

http://www.scielo.br/pdf/cam/v27n3/a05v27n3.pdf https://doi.org/10.1590/S0101-82052008000300005

[12] Sayed, S.M., Elhamahmy, O.O. and Gharib, G.M. (2008) Travelling Wave Solutions for the KdV-Burgers-Kuramoto and Nonlinear Schrödinger Equations Which Describe Pseudospherical Surfaces. Journal of Applied Mathematics, 2008, 576-583.

https://projecteuclid.org/euclid.jam/1234298349 https://doi.org/10.1155/2008/576783

[13] Khater, A.H., Callebaut, D.K. and Sayed, S.M. (2006) Exact Solutions for Some Nonlinear Evolution Equations Which Describe Pseudo-Spherical Surfaces. Journal of Computational and Applied Mathematics, 189, 387-411. https://doi.org/10.1016/j.cam.2005.10.007

[14] Sayed, S.M. and Al-Atawi, N.O. (2017) Hamiltonian Structure, Soliton Solution and Conservation Laws for a New Fifth-Order Nonlinear Evolution Equation Which Describes Pseudo-Spherical Surfaces. American Journal of Computational Mathematics, 7, 166-174. http://www.scirp.org/journal/ajcm https://doi.org/10.4236/ajcm.2017.72015

[15] Stasiewicz, K. and Ekeberg, J. (2008) Dispersive MHD Waves and Alfvenons in Charge Non-Neutral Plasmas. Nonlinear Processes in Geophysics, 15, 681-693. https://www.nonlin-processes-geophys.net/15/681/2008/npg-15-681-2008.html

[16] Raiter, P.D. and Saraykar, R.V. (2013) A Stability Theorem for Large Solutions of Three Dimensional Incompressible Magnetohydrodynamic Equations. IOSR Journal of Mathematics, 7, 62-74.

http://www.iosrjournals.org/iosr-jm/papers/Vol7-issue4/M0746274.pdf?id=6444

[17] Ruderman, M.S. (2002) DNLS Equation for Large-Amplitude Solitons Propagating in an Arbitrary Direction in a High $\beta$ Hall Plasma. Journal of Plasma Physics, 67, 271-276. http://eprints.whiterose.ac.uk/1576/1/rudermanms2.pdf https://doi.org/10.1017/S002237780200168X

[18] Neukirch, T. (1997) Nonlinear Self-Consistent Three-Dimensional Arcade-Like Solutions of the Magnetohydrostatic Equations. Astronomy \& Astrophysics, 325, 847-856. http://citeseerx.ist.psu.edu/messages/downloadsexceeded.html

[19] Petrie, G.J. and Neukirch, T. (1999) Self-Consistent Three-Dimensional Steady State Solutions of the MHD Equations with Field-Aligned Incompressible Flow. Geophysical and Astrophysical Fluid Dynamics, 91, 865-870. https://doi.org/10.1080/03091929908203707

[20] Petrie, G.J., Tsinganos, K. and Neukirch, T. (2005) Steady 2D Prominence-Like Solutions of the MHD Equations with Field-Aligned Compressible Flow. Astronomy \& Astrophysics, 429, 1081-1092. https://doi.org/10.1051/0004-6361:20041706 